\title{
Blanca Hernandez-Ledesma ${ }^{1}$, Chia-Chien Hsieh ${ }^{2}$, and Ben O. de Lumen ${ }^{3}$
}

1 Instituto de Investigación en Ciencias de la Alimentación (CIAL, CSIC-UAM, CEI UAM+CSIC). Nicolás Cabrera, 9. 28049 Madrid, Spain

${ }^{2}$ Department of Human Development and Family Studies (Nutritional Science \& Education), National Taiwan Normal University, No. 162, Heping East Road, Section 1, Taipei, Taiwan

${ }^{3}$ Department of Nutritional Sciences and Toxicology, University of California at Berkeley, 119 Morgan Hall, CA-94720-3104, USA

\section{LUNASIN}

\section{Definition}

Lunasin is a naturally-occurring peptide found in soybean and other plants with potent chemopreventive properties against different types of cancer.

\section{Characteristics}

Lunasin (from the Tagalog word "lunas" for cure) is a 43 amino-acid peptide initially identified in the soybean cotyledon (1). It corresponds to the small subunit peptide encoded within the soybean $2 S$ albumin Gm2S-1 gene that also codes for a methionine-rich protein, a signal peptide, and a linker peptide. The sequence of lunasin ${ }^{1}$ SKWQHQQDSCRKQLQGVNLTPC ${ }^{22}{ }_{2}^{23}$ EKHIMEKIQG $^{32}{ }_{-}^{33} \mathrm{RGD}^{35}$-DDDDDDDD ${ }^{43}$ (National Center for Biotechnology Information, NCBI, accession number AAP62458) is composed of four regions: fragment $\mathrm{f}(1-22)$ without any known function yet; $f(23-32)$ targeting lunasin to chromatin; f(33-35) corresponding to RGD cell adhesion motif internalizing lunasin into the cell nucleus, and a continuous sequence $\mathrm{f}(35-43)$ of nine aspartic acid (D) residues responsible for lunasin’s direct binding to core histones. 
Once identified in soybean, the presence of this peptide has been also demonstrated in other legumes such as lupin, plant species of the Solanaceae family, and cereals such as rye, barley, wheat, oat, triticale, and amaranth. Because of the absence of $2 \mathrm{~S}$ albumins in cereals, recent studies have focused on elucidating the actual origin of lunasin, suggesting that this peptide is synthesized and secreted by microorganisms, either as a mature peptide or a precursor which is then processed by a microbial or endogenous plant proteinase.

The chemopreventive properties of lunasin have been demonstrated in both in vitro and in vivo studies (2). First studies demonstrated that lunasin, in absence of carcinogens, seems not to affect neither cell morphology nor cell proliferation. However, when a transformation event occurs lunasin gets into action within the cell preventing induced-carcinogenic processes. At nanomolar concentrations, lunasin added exogenously significantly suppresses chemical-

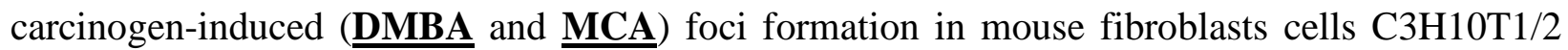
(C3H). This effect is also produced by Lunasin in NIH3T3 cells, being 4-fold more effective (on a molar basis) than the Bowman-Birk inhibitor (BBI), a known cancer-preventive agent from soy. Lunasin also prevents transformation of mammalian cells by viral oncogenes. It inhibits, in a dose-dependent manner, foci formation in C3H cells and NIH3T3 cells transfected with the oncogene E1A, known to induce cell proliferation by inactivating the tumor suppressor retinoblastoma protein $(\mathrm{Rb})$. Lunasin is effective even when added up to 15 days after transfection with E1A gene, suggesting its efficacy when applied even after the transformation event. Lunasin also suppresses colony formation induced by the ras-oncogene in MCF-7 cells stably transfected with an inducible form of the oncogene.

Subsequent studies have proved that lunasin peptide also exerts anti-neoplastic effects in established cancer breast, colon, prostate, leukemia, and lymphoma cells lines. Moreover, efforts demonstrating the in vivo chemopreventive role of this peptide have been designed in chemical 
carcinogen-induced and xenograft animal models. The effects of this peptide are exerted through modulating multiple cellular pathways implied in the stages of initiation, promotion and progression of cancer. In this line, several studies have reported that this peptide is able to modulate expression of different genes and proteins involved in former mentioned characteristics of tumor cells by controlling cell cycle, apoptosis, and signaling transduction. The ability of lunasin to arrest cell cycle at the S- and G2-M phases by increasing the levels of cyclindependent kinases (CDK) inhibitors p15, p21 and p27, down-regulating the cyclins D1, D3, CDK4 and CDK6, and inhibiting the phosphorylation of retinoblastoma protein (Rb) has been demonstrated in transfected fibroblasts, and breast and colon cancer cells. High levels of two proliferation molecular biomarkers, Ki-67 and the proliferating cell nuclear antigen (PCNA) have been found in a variety of malignant cells lines, and have been markedly linked with the aggressiveness and growth capacities of the tumor. Intraperitoneal administration of lunasin in xenograft breast MDA-MB-231 and colorectal KM12L4 cancer liver metastasis mice models has been reported to suppress cell cycle progression through down-regulation of these two factors.

Another mechanism by which lunasin exerts anti-neoplasic effects is through its apoptosis-inducing activity. In colon cancer HT-29 and KM12L4 cells, lunasin modulates the ratio between anti-apoptotic marker $\mathrm{Bcl}-2$ and pro-apoptotic marker Bax, as well as regulates the activity of pro-apoptotic nuclear clusterin, cytochrome c, and caspase-3. In human breast cancer MCF-7 cells, lunasin has been found to increase the expression and protein levels of the tumor suppressor Phosphatase and tensin homolog (PTEN), and to enhance nuclear localization of that protein resulting in the induction of PTEN-mediated cellular apoptosis. Moreover, addition of lunasin to leukemia L1210 cells provoked a notable increase of caspase $-8,-9$, and -3 activities. In breast cancer MDA-MB-231 cells, lunasin acts synergistically with both aspirin and anacardic acid increasing the percentage of early and late-apoptotic cells. 
Histological analysis of stained tumor sections of xenograft MDA-MB-231 mice showed that lunasin-treated animals presented tumor destruction areas replaced by apoptotic and necrotic cells, and significantly higher levels of TUNEL-positive apoptotic cells. In addition, intraperitoneal injection of this peptide to colorectal cancer liver metastasis mice provoked a significant increase of the expression of the pro-apoptotic biomarker Bax, reduction of expression of the anti-apoptotic biomarker Bcl-2, and synergistic effects with oxaliplatin inducing apoptosis through modulation of the Bcl-2:Bax ratio.

An epigenetic mode of action for lunasin has been suggested (Figure 1) (3), indicating that lunasin selectively kills cells that are being transformed by disrupting the dynamics of histone acetylation-deacetylation when a transforming event occurs. Under steady-state conditions in the cell, core histones $\mathrm{H} 3$ and $\mathrm{H} 4$ are mostly in a deacetylated (repressed) state, being the balance between histone acetylation-deacetylation a key cellular process linked to signal transduction pathways including those involved in cancer development. Beyond its internalization into the cell nucleus, biochemical evidence has shown that lunasin, both synthetic and naturally-extracted from plants, is able to bind to deacetylated histones inhibiting their acetylation. Moreover, lunasin has been found to inhibit the acetylation process in cells exposed to sodium butyrate, a known histone deacetylase inhibitor, and to compete with different histone acetyltransferases (HATs) such as PCAF, CBP/p300 and yGCN5. The tumor suppressor protein $\mathrm{Rb}$ functions by interacting with E2F promoter and recruiting histone deacetylases (HDAC) to keep the core histones in the deacetylated (repressed) state. The inactivation of Rb by the oncoprotein E1A dissociates the Rb-HDAC complex, exposing the deacetylated core histones to be acetylated by the action of HATs. When this event occurs, lunasin is triggered into action and binds to the deacetylated core histones, turning off transcription, which is perceived as abnormal by the cell and commits apoptosis. This epigenetic mechanism shows that lunasin can influence 
regulatory pathways involving chromatin modifications that may be fundamental to carcinogenic pathways in general.

Recently, antioxidant and anti-inflammatory properties of lunasin have been described which might contribute on its chemopreventive effects. In vitro, lunasin has been found to act as a potent scavenger of superoxide and peroxil radicals, inhibitor of linoleic acid oxidation and blocking agent of hydroxyl radical generation by chelating iron ferrous ions, protecting DNA from induced-oxidative damage. These antioxidant properties have been confirmed by cell culture models. The production of reactive oxygen species (ROS) has been reported to decrease in lipopolysaccharide (LPS)-induced RAW264.7 macrophages after treatment with lunasin. Also, this peptide is able to improve cell viability and decrease ROS levels in intestinal Caco-2 and liver HepG2 cells challenged by hydrogen peroxide and tert-butylhydroperoxide. In latter cells, lunasin also provokes depletion of reduced glutathione, modulation of glutathione peroxidase and catalase activities, decrease of carbonyl groups and recovery from cell death by restraining the caspase-3 apoptotic pathway.

Lunasin has been also demonstrated to inhibit, in the LPS-induced RAW 264.7 murine macrophage cellular model, pro-inflammatory mediators such as interleukin (IL)-6, IL-1 $\beta$, and tumor necrosis factor (TNF)- $\alpha$ by suppression of cyclooxygenase (COX)-2/prostaglandin (PG) $\mathrm{E}_{2}$, inducible nitric oxide synthase (iNOS)/nitric oxide (NO) and nuclear factor-kappa B (NF-кB) pathways. In this cell model, lunasin has been found to affect the expression of genes involved in cellular growth and proliferation, cellular function, cell to cell signaling, and cell cycle.

Bioavailability is an important feature of an ideal cancer preventive agent. To exert this effect, once orally ingested, the peptides have to survive degradation by gastrointestinal and serum proteinases and peptidases, reaching the target tissue or organ in an active form. In vitro bioavailability studies simulating gastrointestinal digestion have demonstrated that lunasin, both 
synthetic as well as purified from food is markedly hydrolyzed by pepsin and pancreatin; however, lunasin in foods has been shown to resist the action of these enzymes. Naturally present protease inhibitors in foods, such as BBI and Kunitz trypsin inhibitor (KTI), have been found to be major responsible for the lunasin's protection against gastrointestinal enzymes. In vivo bioavailability studies carried out with animals and humans have also confirmed above in vitro findings. Lunasin is bioavailable after being orally administrated to mice and rats, being absorbed through gastrointestinal barrier and extensively distributed reaching different tissues such as liver, kidney, lung, mammary gland, prostate, and brain, where lunasin exerts its bioactive properties. In humans, an average of $4.5 \%$ absorption of lunasin from the total ingested in $50 \mathrm{~g}$ of soy protein has been reported.

Recently, accumulating evidence has shown that lunasin not only executes a chemopreventive role and possesses inherent antioxidative, anti-inflammatory properties, but also is involved in cholesterol biosynthesis, and central nervous system and immune system regulation. Lunasin's multiple bio-functions make it a promising peptide agent in the prevention/treatment of different disorders.

\section{References}

(1) Galvez AF, de Lumen BO. (1999) A soybean cDNA encoding a chromatin-binding peptide inhibits mitosis of mammalian cells. Nature Biotechnology 17: 495-500.

(2) Fernández-Tomé S, Hernández-Ledesma B. (2015). An update on lunasin research, a bioactive seed peptide for health promotion. In: Govil JN (Ed) Recent progress in medicinal plants. Studium Press LLC, TX, USA, in press.

(3) de Lumen BO (2005) Lunasin: a cancer-preventive soy peptide. Nutrition Reviews 63:16-21. 


\section{Figure 1.}

E1A-Rb-HDAC model to explain the ability of Lunasin to suppress E1A-induced transformation. Top diagram: Rb controls G1/S transcription by interacting with E2F promoter and recruiting HDAC to keep the core histones in the deacetylated (repressed) state. In a cell being transformed, E1A inactivates Rb and dissociates Rb-HDAC complex, exposing the deacetylated core histones in the E2F promoter (medium diagram). Lunasin competes with histone acetyltransferase (HAT) in binding to the deacetylated core histones. Bottom diagram: HAT binds and acetylates core histones, turning on E2F cell cycle transcription factors, leads to cell proliferation. Lunasin binds, turns off the transcription, perceived as abnormal by cell, results in apoptosis. 


\section{Cross-references}

\section{Histones}

Highly alkaline proteins found in the nucleus of eukaryotic cells responsible for packaging and ordering the DNA into structural units called nucleosomes. They are the chief protein components of chromating, playing a crucial role in gene regulation.

\section{DMBA}

7,12-Dimethylbenz[a]anthracene. Polycyclic aromatic hydrocarbon with carcinogenic, tumorigenic and teratogenic effects.

\section{MCA}

3-methylcholanthrene. Polycyclic aromatic carcinogen compound often used to study the effects of anticancer compounds.

\section{Bowman-Birk inhibitor (BBI)}

Serine protease inhibitor consisting of a single chain of 71 amino acid residues cross-linked by seven pairs of disulfide bonds and a well characterized ability to inhibit trypsin and chymotrypsin. It has been shown to be capable of preventing or suppressing carcinogenic processes in a wide variety of in vitro and in vivo animal model systems.

\section{Retinoblastoma protein}

Tumor suppressor protein belonging to the pocket protein family whose members have a pocket for the functional binding of other proteins. One of its major functions is the prevention of excessive cell growth by inhibiting cell cycle progression until the cell is ready to divide. When the cell is ready, Rb is phosphoryltated, becoming inactive, thus allowing cell cycle progression. It also acts as a recruiter of several chromatin remodeling enzymes such as methylases and acetylases. 


\section{Cyclin-dependent kinases (CDK)}

Family of protein kinases with molecular weights ranged from 34 to $40 \mathrm{kDa}$, present in all know eukaryotics, that bind a regulatory protein called cyclin. They are involved in the regulation of cell cycle, transcription, mRNA processing and differentiation of nerve cells.

\section{Cyclins}

Family of proteins that control the progression of cells through the cell cycle by activating CDK enzymes.

\section{Phosphatase and tensin homolog (PTEN)}

Human phosphatidylinositol-3,4,5-trisphosphate 3-phosphatase encoded by the PTEN gene. It negatively regulates intracellular levels of phosphatidylinositol-3,4,5-triphosphate in cells and functions as a tumor suppressor by negatively regulating Akt/PKB signaling pathway.

\section{Epigenetic}

Study, in the field of genetics, of cellular and phenotypical variations caused by external or environmental factors that switch genes on and off affecting the mode how cells "read" genes. Epigenetic research describes dynamic alterations in the transcriptional potential of a cell.

\section{Histone acetyltransferases (HATs)}

Family of proteins that acetylate conserved lysine amino acids on histone proteins by transferring an acetyl group from acetyl $\mathrm{CoA}$ to lysine to form $\varepsilon$-N-acetyl lysine. Lysine acetylation neutralizes the positive charge normally present, thus reducing affinity between histone and (negatively charged) DNA which renders DNA more accessible to transcription factors. Also, histone acetylation and other posttranslational modifications generate binding sites for specific protein-protein interaction domains. 


\section{Kunitz Trypsin inhibitor (KTI)}

Antinutritional/allergen protein found in soybean that acts as an inhibitor of pancreatin and chymotrypsin. 